\title{
Analyzing the Performance of Interacting Spherical Tank System using Internal Model Controller (IMC) and Metaheurstic Algorithm
}

\author{
K. Krishnapriya ${ }^{1}$, M. Rathina Devi ${ }^{2}$, U. Roshini ${ }^{3}$, Dr. A. Jayachitra ${ }^{4}$ \\ Department of Instrumentation and Control Engineering, Sri Manakula Vinayagar Engineering College e $^{1,2,3,4}$
}

\begin{abstract}
One of the major problems in process industries is to control the liquid level in a tank. Proportional Integral Derivative (PID) controllers are commonly used controller in the process industries. Interacting Spherical Tank Liquid Level System (ISTLLS) has the characteristics of nonlinearity due to the dynamic behavior and area of cross section of tank. The time delay reduces the performance of the system that occurs when conventional controller is used. Our project aim is to analyze the performance of the Internal Model Control (IMC) based PID Controller and Metaheurstic algorithm for an Interacting spherical tank system. Using the mathematical model of the process simulation is done and their performance is analyzed under MATLAB environment. Metaheurstic algorithm like Genetic Algorithm (GA) and Firefly Algorithm (FA) are used for obtaining the best PID tuning values. The motive of Metaheurstic algorithm is to provide the auto tuning and obtaining the best solution to reduce error. Finally the performance of conventional controller (PID), Internal Model Control (IMC)-PID controller, FA-PID algorithm and GA-PID algorithm performances are compared and analyzed.
\end{abstract}

Keywords: Nonlinear system, Genetic Algorithm Optimization, and Firefly Algorithm Optimization, IMC PID Controller.

\section{INTRODUCTION}

Generally, nonlinear problems are problematic to solve and are not easily understandable than that of linear problems. The control of liquid level in the tanks and the flow between the tanks are the two basic problems in the process industries. In process industries the liquids are pumped and stored in tanks and thereafter pumped to next tank. The advantages of Spherical Tank are inexpensive and efficient washing, intensified production and reduced product loss. The spherical Tank is widely used in various industries like petrochemical industries, paper making industry, water treatment industries.IMC PID controller reduces the peak overshoot and will give high rise time.

IMC Controllers are widely recognized in process industries due to having only single tuning parameter $(\lambda)$.

Tuning parameter $(\lambda)$ is always depends on the time constant and delay time.

Generally IMC tuning controller is used to approximate dead time which is essential need for delay dominated systems. IMC controller provides different tuning techniques to control the level of water in highly nonlinear systems. Usually Proportional Integral (PI) controllers required to meeting the optimal response in linear systems and PID controllers are required to meet desired response in nonlinear systems. Nonlinear systems are the delay dominated systems with unstable characteristics. Unstable processes can be easily tuned by using IMC tuning methods

\section{PROCESS DESCRIPTION}

The Two Tank Spherical Interacting System (TTSIS) shown in Fig.1, is a highly nonlinear system and designing of controller is a challenging task. TTSIS consists of two identical spherical tanks(TANK1 and TANK2), two independent pumps that deliver the liquid flows FIN1 and FIN2 to TANK1 and TANK2 through the two control valves CV1 and CV2 respectively. These two tanks are interconnected at the bottom of the tank through a manually controlled valves MV12 whose valve co-efficient is $\beta 12$.Fout1 and Fout2 are the two output flows from TANK1 and TANK2 respectively. These flows are adjusted through manually controlled valves MV1 and MV2 with valve coefficient $\beta 1$ and $\beta 2$ respectively. The operating parameters of TTSIS are shown in table I

Where,

$\rho=$ density

FIN $=$ Volumetric flow rate for inlet stream

FOUT $=$ Volumetric flow rate for outlet stream 
$A=$ Area of the spherical tank with respect to Change the flow $\mathrm{h} 1, \mathrm{~h} 2=$ Height of spherical tank 1 and 2

$\mathrm{dh} / \mathrm{dt}=$ Change in height of liquid level

Table 1.1 Parameters values of spherical tank process

\begin{tabular}{|l|l|l|}
\hline Terms used & Terms description & Values substituted \\
\hline $\mathrm{F}_{\text {in }}$ & Input flow & 107.25 \\
\hline$\beta_{1}$ & Control valve for tank 1 & 78.28 \\
\hline$\beta_{2}$ & Control valve for tank 2 & 19.69 \\
\hline $\mathrm{h}_{1}$ & Height of the tank 1 & 30 \\
\hline $\mathrm{h}_{2}$ & Height of the tank 2 & 30 \\
\hline $\mathrm{C}_{1}$ & Capacity of the tank 1 & 0.3267 \\
\hline $\mathrm{R}_{1}$ & Radius of the tank 1 & 0.03522 \\
\hline $\mathrm{C}_{2}$ & Capacity of the tank 2 & 0.09128 \\
\hline $\mathrm{R}_{2}$ & Radius of the tank 2 & 0.5564 \\
\hline
\end{tabular}

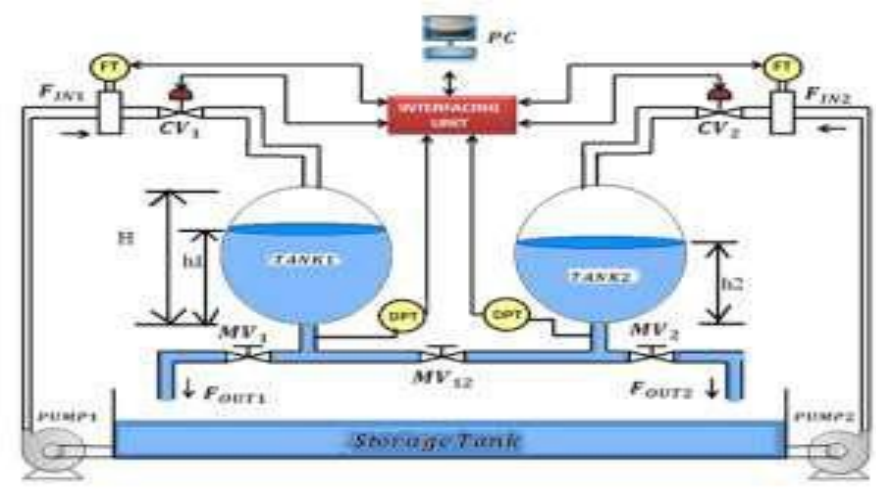

fig 1.1 Schematic of TTSIS

The model of Tank 1 can be represented as,

Taking linearization,

$$
A_{1} h_{1} \frac{d h_{1}}{d t}=F_{i n}-\beta \sqrt{h_{1}-h_{2}}
$$

$$
A_{1} h_{1} \frac{d\left(\partial h_{1}\right)}{d t}=F_{1}\left(F_{i n 1}\right)-F_{1}\left(h_{1}-h_{2}\right)
$$

Applying partial difference,

$$
A_{1} h_{1} \frac{d\left(\partial h_{1}\right)}{d t}=\left(\frac{\partial F_{1}}{\partial F_{i n 1}}\right) \partial F_{i n}-\left(\frac{\partial F_{2}}{\partial h_{2}}\right) \partial h_{1}-\left(\frac{\partial f_{2}}{\partial h_{2}}\right) \partial h_{2}
$$

On rearranging,

$$
A_{1} h_{1} \frac{d\left(\partial h_{1}\right)}{d t}=\partial F_{i n 1}-\frac{\beta_{12}}{2 \sqrt{h_{1}-h_{2}}} \partial h_{1}+\frac{\beta_{12}}{2 \sqrt{h_{1}-h_{2}}} \partial h_{2}
$$

Apply Laplace transform..,

$$
\partial h_{1}=\frac{\partial F_{i n 1}+\frac{\beta_{12}}{2 \sqrt{h_{1}-h_{2}}} \partial h_{2}}{A_{1} h_{1}(s)+\frac{\beta_{12}}{2 \sqrt{h_{1}-h_{2}}}}
$$


The model for tank 2,

$$
A_{1} h_{1} \frac{d h_{2}}{d t}=\beta_{12} \sqrt{h_{1}-h_{2}}-\beta_{2} \sqrt{h_{2}}
$$

Taking linearization..,,

$$
A_{2} h_{2} \frac{d\left(\partial h_{2}\right)}{d t}=F_{1}\left(h_{1}-h_{2}\right)-F_{2}\left(h_{2}\right)
$$

Apply partial difference...,,

$$
A_{2} h_{2} \frac{d\left(\partial h_{2}\right)}{d t}=\left(\frac{\beta_{12}}{2 \sqrt{h_{1}-h_{2}}} \partial h_{1}-\frac{\beta_{12}}{2 \sqrt{h_{2}-h_{2}}} \partial h_{2}-\frac{\beta}{2 \sqrt{h_{2}}} \partial h_{2}\right)
$$

Apply Laplace transform..,,

$$
\begin{aligned}
& \left(A_{2} h_{2}(s)+\frac{\beta_{12}}{2 \sqrt{h_{1}-h_{2}}}+\frac{\beta_{2}}{2 \sqrt{h_{s}}}\right) \partial h_{2}-\frac{\beta_{12}}{2 \sqrt{h_{1}-h_{2}}} \partial h_{1}=\frac{\beta_{12}}{2 \sqrt{h_{1}-h_{2}}} \frac{\left[\partial F_{i 11}+\frac{\beta_{12}}{2 \sqrt{h_{1}-h_{2}}} \partial h_{2}\right]}{A_{1} h_{1}(s)+\frac{\beta 12}{2 \sqrt{h_{1}-h_{2}}}} \\
& \left.A_{2} h_{2}(s)+\frac{\beta_{12}}{2 \sqrt{h_{1}-h_{2}}}+\frac{\beta_{2}}{2 \sqrt{h_{s}}}\right) \partial h_{2}-\frac{\beta_{12}}{2 \sqrt{h_{1}-h_{2}}} \partial h_{1}
\end{aligned}
$$

Let us assume......,

$$
\begin{aligned}
& C_{1}=\frac{1}{2 \sqrt{h_{1}-h_{2}}} \\
& C_{2}=\frac{1}{2 \sqrt{h_{2}}} \\
& R_{1}=\frac{1}{\beta_{12} C_{1}} \\
& R_{2}=\frac{1}{\beta_{2} C_{2}}
\end{aligned}
$$

Substitute $\mathrm{R}_{1}, \mathrm{R}_{2}, \mathrm{C}_{1}, \mathrm{C}_{2}$ in the equation 3.11

$$
\frac{\partial h_{2}}{\partial F_{i n 1}}=\frac{R_{2}}{\tau_{1} \tau_{2} S^{2}\left(\tau_{1}+\tau_{2}+A\left(h_{1}\right) R_{2}\right) S+1}
$$

By substituting the values $\mathrm{R}_{1}, \mathrm{R}_{2}, \mathrm{C}_{1}, \mathrm{C}_{2}$ in the equation 3.11 the corresponding transfer function is obtained as given in equation 3.12

$$
\mathbf{G}(\mathbf{s})=\frac{H_{2}(s)}{Q_{i}(s)}=\frac{0.1317}{\left(68.39 s^{2}+68.60 s+1\right)} e^{-4 s}
$$

The second order system has been reduced to first order using

$$
\frac{0.68}{(69 s+1)} e 4 s
$$

$\mathrm{G}(\mathrm{s})=$ 
Vol. 6, Issue 4, April 2017

\section{IMC BASED PID CONTROLLER}

The IMC design procedure is a two-step design process that aims to provide a suitable trade-off between performance and robustness. In Step 1 a stable and causal controller is obtained that is optimal with respect to either the integral of squared error (ISE) or integral of absolute error (IAE) criteria for step changes to the control system; the second step augments the controller from Step 1 with a filter to insure that the IMC controller is proper. For many simple processes of interest the IMC controller, when implemented in classical feedback form, leads to a PID-type controller. Various illustrative examples are developed and evaluated under a common setting.

The performance of IMC-based PID controller tuning for systems with delay is examined and contrasted with popular classical Ziegler-Nichols and Cohen-Coon PID controller tuning rules. The IMC structure is rearranged to get a standard feedback control system so that open loop unstable system can be handled. This is done to improve the input disturbance rejection. The IMC based PID structure uses the process model as in IMC design. In the IMC procedure the controller $\mathrm{Qc}(\mathrm{s})$ is directly based on the invertible part of the process transfer function. The IMC results in only one tuning parameter which is filter tuning factor but the IMC based PID tuning parameters are the functions of this tuning factor. The selection of the filter parameter is directly related to the robustness. IMC based PID procedures uses an approximation for the dead time. And if the process has no time delays it gives the same performance as does the IMC

\section{GENETIC ALGORITHM}

Genetic algorithms (GA) are an evolutionary optimization approach, which are an alternative to traditional optimization methods. GA is one of the most appropriate methods for complex non-linear models where location of the global optimum is a difficult task. GA follows the concept of solution evolution by stochastically developing generations of solution populations using a

given fitness. Genetic algorithms are a probabilistic search approach, which are founded on the ideas of evolutionary processes. The GA procedure is based on the Darwinian principle of survival of the fittest. An initial population is created containing a predefined number of individuals, each represented by a genetic string. Each individual has an associated fitness measure. The concept that the fittest best individuals in a population will produce a fitter offspring is then implemented in order to reproduce the next population. Selected individuals are chosen for reproduction at each generation, with an appropriate mutation factor to randomly modify the genes of an individual, in order to develop the new population. The result is another set of individuals based on the original subjects leading to subsequent populations with better fitness and those with lower fitness

will naturally get discarded from the population; For most applications of genetic algorithms to optimization problems, the real coding technique is used to represent a solution to a given problem. In real coding implementation, each chromosome is encoded as a vector of real numbers, of the same lengths as the solution vector. According to control objectives, three parameters $\mathrm{Kp}, \mathrm{Ki}$ and $\mathrm{K}_{\mathrm{d}}$ of a PID controller are required to be designed in this research. For a given problem with three decision variables, this paper adopts a real-valued vector [x1, x2, x3] as a chromosome to represent a solution to the problem.

\section{FIREFLY ALGORITHM}

The Firefly Algorithm was inspired by the flashing nature of fireflies. There are over 2000 species of fireflies, most of which produce a bioluminescence from their abdomen. Each species of firefly produces its own pattern of flashes, the main purpose for their flashing is to attract a mate. Bioluminescence is the ability of certain animals to produce light. Two fundamental functions of such flashes are to attract mating partners and potential prey. The rhythmic flash, the rate of flashing and the amount of time form part of the signal system that brings both sexes together. Females respond to a male's unique pattern of flashing in the same species, while in some species such as postures, female fireflies can mimic the mating flashing pattern of other species, so as to attract and eat the male fireflies who may mistake the flashes as a potential suitable The flashing can also be used to send information between fireflies. The idea of this attractiveness and information passing leads to the inspiration for FA.

For a given medium with a fixed light absorption coefficient ' $\gamma$ ', the light intensity ' $I$ ' varies with distance ' $r$ ' monotonically and exponentially.

$$
\mathrm{I}=\mathrm{I}_{\mathrm{o}} \mathrm{e}^{-\gamma \mathrm{r}}
$$

The distinctive attractiveness ' $\beta$ ' of a firefly.

$$
\beta=\beta_{\mathrm{o}} \mathrm{e}^{-\gamma \mathrm{r}^{2}}
$$

The movement of firefly ' $\mathrm{i}$ ' is attracted to another more attractive (brighter) firefly ' $\mathrm{j}$ ' as determined by. 


$$
\mathrm{x}_{\mathrm{i}}=\mathrm{x}_{\mathrm{i}}+\beta_{\mathrm{o}} \mathrm{e}^{-\gamma \mathrm{r}_{\mathrm{i}, \mathrm{j}}^{2}}\left(\mathrm{x}_{\mathrm{j}}-\mathrm{x}_{\mathrm{i}}\right)+\alpha(\text { rand }-0.5)
$$

where, the first term $x_{i}$ is the current position of a firefly, the second term $\beta_{o} e^{\gamma r_{i, j}^{2}}\left(x_{j}-x_{i}\right)$ is the attractiveness factor and the last term $\alpha($ rand-0.5) allows random movement of firefly.

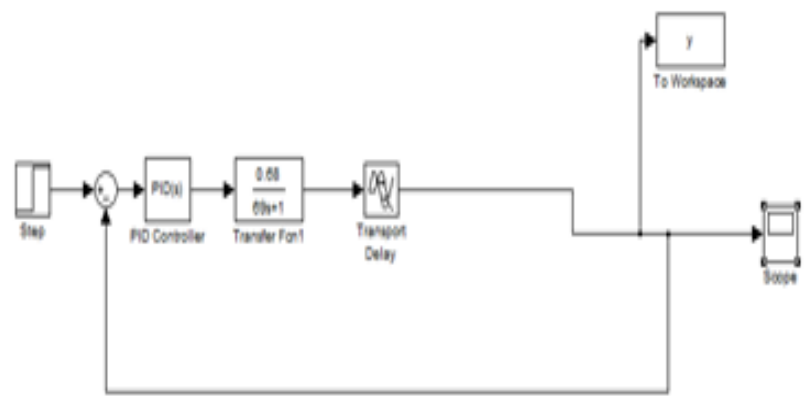

Fig 1.2 simulink block of IMC based PID controller

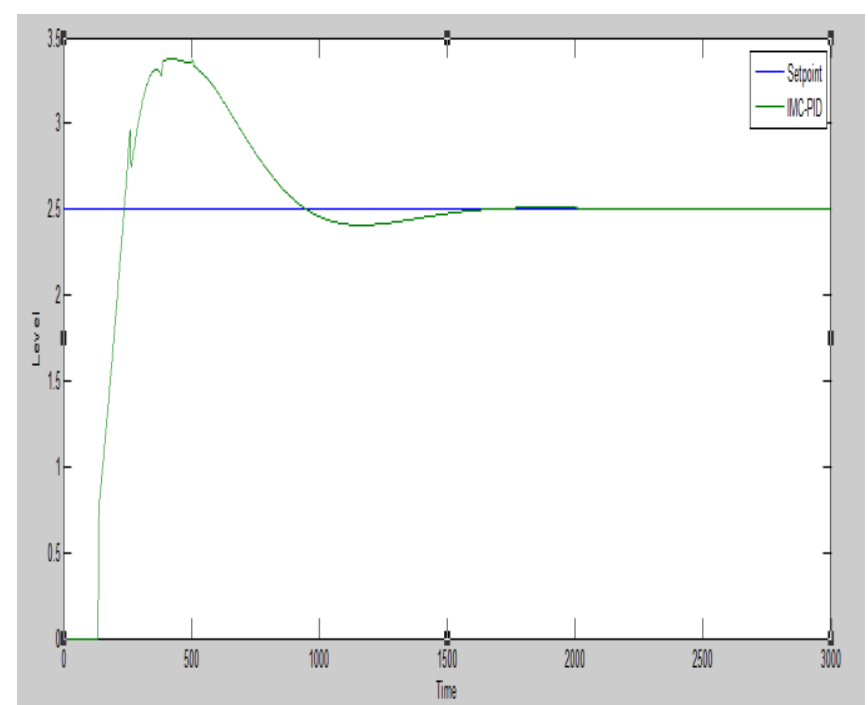

Fig 1.3 Response obtained for IMC based PID controller

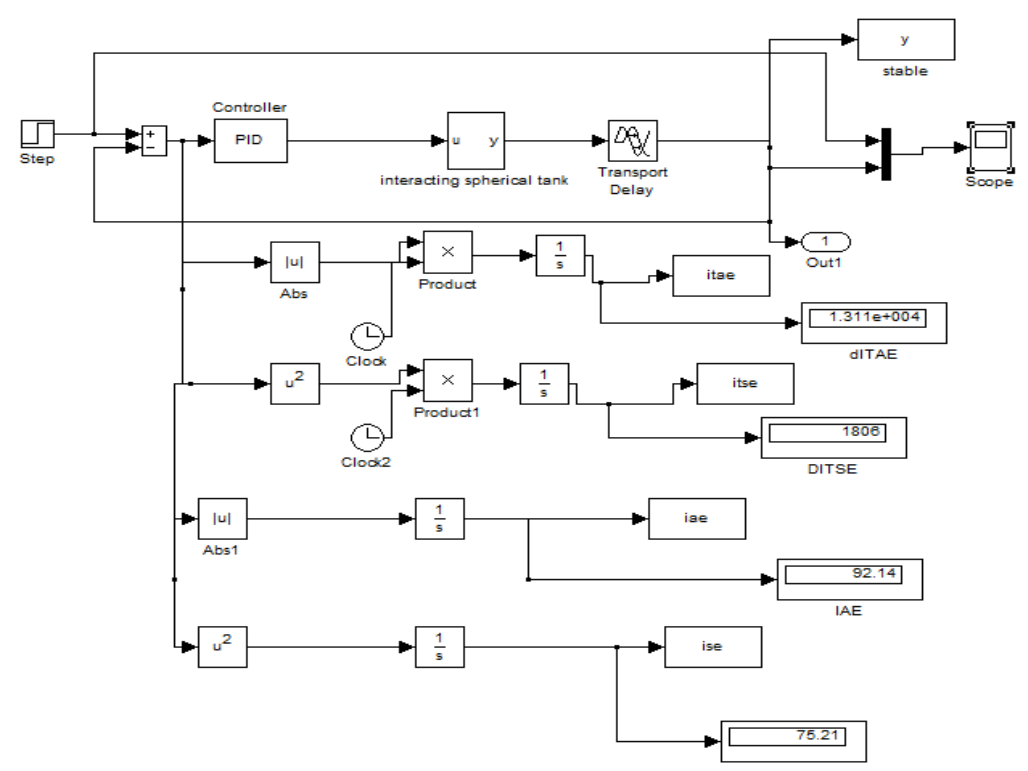

Fig 1.4 Simulink Block for Firefly Algorithm 
International Journal of Advanced Research in Computer and Communication Engineering ISO 3297:2007 Certified

Vol. 6, Issue 4, April 2017

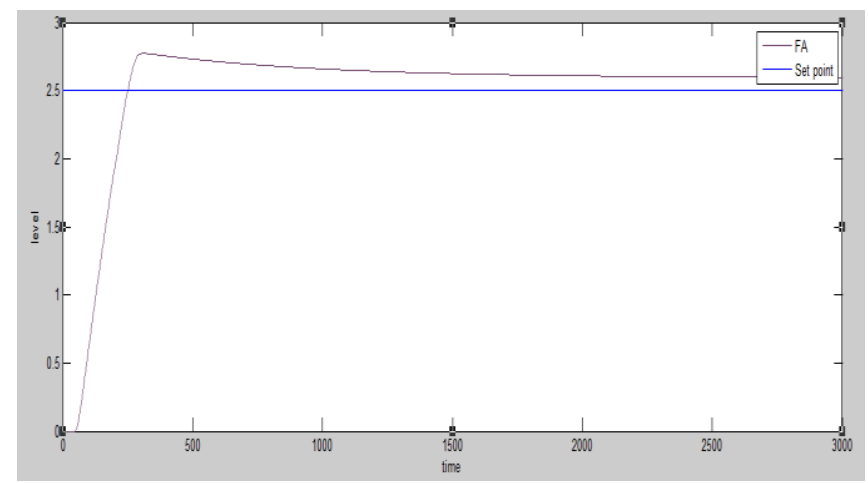

Fig 1.5 Response obtained for Firefly Algorithm

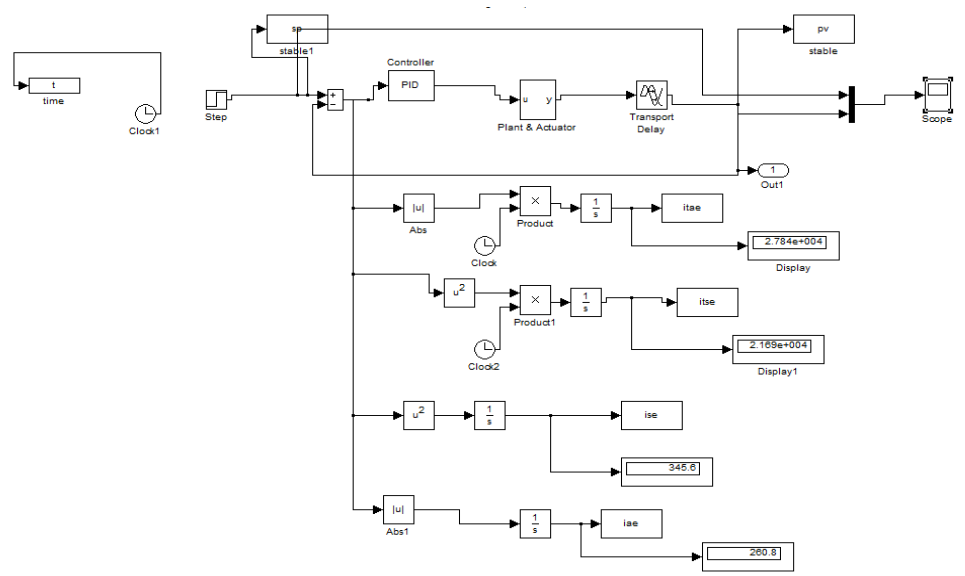

Fig 1.6 Simulink block for Genetic Algorithm

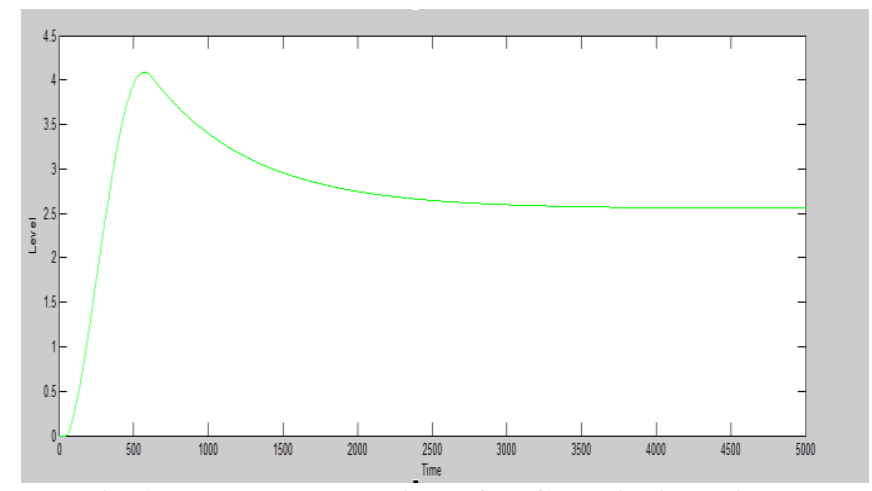

Fig 1.7 Response obtained for Genetic Algorithm

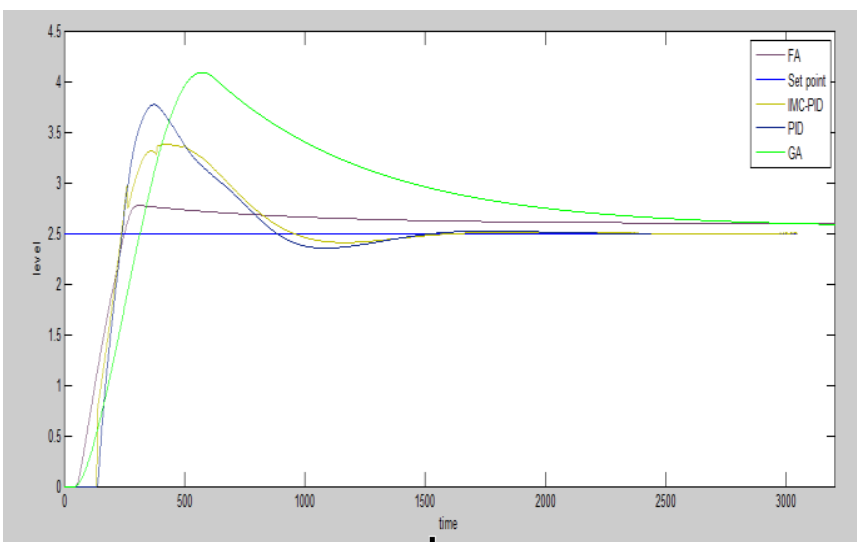

Fig 1.8 Comparison of IMC-PID, FA-PID and GA-PID 
Vol. 6, Issue 4, April 2017

TABLE 1.2 Performance Indices for the considered Controllers

\begin{tabular}{|l|l|l|l|l|}
\hline Controller & IAE & ISE & ITAE & ITSE \\
\hline IMC based PID & $\mathbf{4 0 . 7 1}$ & $\mathbf{3 8 . 6}$ & $\mathbf{3 6 8 9}$ & $\mathbf{2 7 5 . 7}$ \\
\hline Firefly Algorithm & $\mathbf{9 4 . 7 5}$ & $\mathbf{7 6 . 2}$ & $\mathbf{1 . 3 4}$ & $\mathbf{1 . 8 9 7}$ \\
\hline Genetic Algorithm & $\mathbf{2 6 0 . 8}$ & $\mathbf{3 4 5 . 5}$ & $\mathbf{2 . 7 8 4}$ & $\mathbf{2 . 1 6 9}$ \\
\hline
\end{tabular}

\section{CONCLUSION}

IMC- PID, Firefly Algorithm based PID and Genetic Algorithm based PID controllers are designed for Interacting spherical two tank system. Performance analysis has been done using performance Indices like Integral Time Absolute Error (ITAE), Integral Square Error (ISE), Integral Time Square Error (ITSE) and Integral Absolute Error (IAE). From the analysis it is inferred that the ITAE error of IMC- PID controller is large and he optimization algorithm (FA) based PID controller has less error value.

\section{REFERENCES}

1. MiralChangela, Ankit Kumar, “To Review On Liquid Level System Using P,Pi And Pid Controller”,National Conference on Emerging Trends in Computer, Electrical \& Electronics (ETCEE-2015) International Journal of Advance Engineering and Research Development (IJAERD)

2. K.Harikrishnaa, J.SatheeshKumarb and MahaboobShaikc, "Design and Development of Model Based Controller for a Spherical Tank",International Journal of Current Engineering and Technology,ISSN 2277 - 4106

3. D.Dinesh Kumar, C.Dinesh and S. Gautham "Design and implementation of skogestad PID controller for Interacting Spherical Tank" International Journal of Advanced Electrical and Electronics Engineering (IJAEE) volume -2 Issue-4, 2013

4. M. Kalyan chakravarthi Pannem K.Vinay and Nithya Venkatesan "Design and Simulation of Internal Model Controller for Real Time Nonlinear"

5. D.Pradeepkannan and Dr.S.Sathiyamoorthy "Control of a Nonlinear Spherical Tank Process Using GA Tuned PID Controller" International Journal of Innovative Research in Science, Engineering and Technology Volume 3, Special Issue 3, March 2014

6. MiralChangela, Ankit Kumar, “To Review On Liquid Level System Using P,Pi And Pid Controller",National Conference on Emerging Trends in Computer, Electrical \& Electronics (ETCEE-2015) International Journal of Advance Engineering and Research Development (IJAERD)

7. K.Harikrishnaa, J.SatheeshKumarb and MahaboobShaikc, "Design and Development of Model Based Controller for a Spherical Tank",International Journal of Current Engineering and Technology,ISSN $2277-4106$

8. D.Dinesh Kumar, C.Dinesh and S. Gautham "Design and implementation of skogestad PID controller for Interacting Spherical Tank" International Journal of Advanced Electrical and Electronics Engineering (IJAEE) volume -2 Issue-4, 2013

9. M. Kalyan chakravarthi Pannem K.Vinay and Nithya Venkatesan "Design and Simulation of Internal Model Controller for Real Time Nonlinear Process" Indian Journal of Science and Technology (IJST) volume - 8 Issue-18, 2015

10. N.NithyaRani, Dr.S.M.GirirajKumar, and Dr.N.Anantharaman "Modelling and control of temperature process using Genetic Algorithm" International Journal of Advanced Research in Electrical, Electronics and Instrumentation Engineering(IJAREEIE) volume - 2 Issue-11, 2013 